\section{Auto-relato de diagnóstico médico de asma e transtornos mentais comuns entre funcionários de uma universidade no Rio de Janeiro, Brasil: Estudo Pró-Saúde}

\author{
Self-reported history of physician-diagnosed \\ asthma and common mental disorders among \\ civil servants at a public university in Rio \\ de Janeiro, Brazil: the Pró-Saúde Study
}

Katia T. Nogueira 1,2

Claudia S. Lopes 2

Eduardo Faerstein 2

\section{Núcleo de Estudos da Saúde do Adolescente, Universidade do Estado do Rio de Janeiro, Rio de Janeiro, Brasil. 2 Instituto de Medicina Social, Universidade do Estado do Rio de Janeiro, Rio de Janeiro, Brasil. \\ Correspondência C. S. Lopes \\ Departamento de Epidemiologia, Instituto de Medicina Social, Universidade do Estado do Rio de Janeiro. \\ Rua São Francisco Xavier 524 70 andar, Rio de Janeiro, RJ 20559-900, Brasil. lopes@ims.uerj.br}

\begin{abstract}
This study investigates the association between history of asthma and common mental disorders among employees at a public university in the State of Rio de Janeiro, Brazil. Phase 1 cross-sectional data from a cohort study (the Pró-Saúde Study) were collected from 4,030 employees. Asthma was ascertained by self-reported medical diagnosis, and the occurrence of common mental disorders was based on the General Health Questionnaire (GHQ-12). Generalized linear models were used to calculate prevalence rates. Asthma prevalence was 11\% (444), of whom 39.7\% (176) presented common mental disorders. History of asthma was associated with higher income ( $p=$ $0.01)$ and female gender $(p=0.01)$. The analysis adjusted by gender, age, and per capita income revealed an association between asthma and common mental disorders $(P R=1.37 ; 95 \% C I$ : 1.22-1.55). Employees with less than 10 years since their asthma diagnosis showed a higher prevalence of common mental disorders $(P R=$ 1.88; 95\%CI: 1.32-2.70). These findings suggest that multidisciplinary teams should consider emotional aspects of asthma patients, especially those recently diagnosed.
\end{abstract}

Asthma; Mental Disorders; Cohort Studies; Questionnaires

\section{Introdução}

Nas últimas décadas, a prevalência de asma tem aumentado consideravelmente em muitos países. Estima-se que aproximadamente 100 a 150 milhões de pessoas sofram de asma no mundo todo, e cerca de 180 mil óbitos anuais ocorram devido à doença ${ }^{1}$. Estudo multicêntrico (International Study for Asthma and Allergies in Childhood - ISAAC) realizado em 56 países mostrou uma variabilidade na prevalência de asma ativa na infância de 1,6 a 36,8\%, estando o Brasil em oitavo lugar, com a prevalência média de $20 \% 2$. Anualmente, ocorrem cerca de 350 mil internações por asma no Brasil, o que a coloca como quarta causa de hospitalização pelo Sistema Único de Saúde (SUS) - 2,3\% do total - e a terceira causa entre crianças e adultos jovens 3 .

Diferentes estudos têm demonstrado que o aumento da poluição do ar, a prevalência de infecções respiratórias, condições sócio-econômicas precárias, a falta de informações sobre a doença e fatores emocionais podem estar envolvidos no desenvolvimento da asma ${ }^{3,4}$. A chamada "hipótese higiênica" que relaciona o aumento na prevalência de asma com o aumento da higiene e asseio, uso de antibióticos e vacinas, estabelece um elo etiológico entre o aumento da asma nos países industrializados ocidentais e o declínio da incidência das doenças infecciosas, revelando assim transformações significativas na compreensão da etiologia da doença 5 . A relação 
entre fatores emocionais e asma tem sido objeto de investigação de vários estudos. Grande parte desta literatura tem mostrado que os transtornos emocionais podem desencadear sintomas, agravar as crises e interferir na adesão ao tratamento ${ }^{6,7,8}$. Gillaspy et al. ${ }^{9}$ observaram que os adolescentes com diagnóstico de asma auto-referida apresentavam maior risco de transtornos mentais comuns do que os adolescentes sem esse diagnóstico. Entretanto, a possibilidade de causalidade bidirecional não deve ser descartada, e alguns estudos têm mostrado que tais transtornos podem ser também uma conseqüência da asma 9,10, especialmente em decorrência da piora da qualidade de vida em pacientes com asma crônica. Castro et al. 11, em estudo conduzido em população ambulatorial em São Paulo, Brasil, observaram que $63,4 \%$ dos pacientes com asma apresentavam fenômenos depressivos; desses, $43,3 \%$ apresentavam depressão reativa. Foi observado também que os pacientes deixavam de realizar certas atividades e de freqüentar lugares públicos, isolando-se socialmente. Para o autor, a depressão é uma conseqüência do prejuízo da qualidade de vida e produtividade causadas pela asma grave. Em estudo realizado por Machado et al. 12, também no Brasil, a depressão foi observada em $25 \%$ dos pacientes asmáticos, freqüência duas vezes superior à observada em pacientes ambulatoriais e cinco vezes maior do que a da população geral 13,14.

Vale ressaltar que a presença de transtornos mentais, como ansiedade e depressão, vem sendo associada à subestimação da gravidade de crises de asma, favorecendo sua morbidade e letalidade. Estudos sobre a mortalidade por asma têm demonstrado, particularmente entre adolescentes, que os transtornos emocionais constituem também importantes fatores de risco para uma maior letalidade 4,9 .

O primeiro grande estudo em epidemiologia respiratória que utilizou um questionário estruturado foi o Medical Research Council (MRC), na Inglaterra 14 . O questionário utilizado neste estudo - MRC - estimulou o desenvolvimento de outros questionários, como o da Comunidade Européia de Aço e Carvão (ECSC), o questionário de sintomas respiratórios da Sociedade Americana de Tórax e da Divisão de Doenças Pulmonares, assim como o International Union Against Tuberculosis and Lung Disease (IUATLD) 16,17. Em razão da dificuldade no diagnóstico de asma, a validação de novos questionários pode ser realizada comparando-se suas respostas com os resultados da prova de função respiratória, com achados clínicos, ou com resultados obtidos com os questionários clássicos como o MRC. Quando se considera o diagnóstico clínico como padrão- ouro, observa-se que o auto-relato de diagnóstico médico de asma apresenta uma sensibilidade de $68 \%$ e especificidade de $99 \%$ 18. Em estudos epidemiológicos, o uso de questionários com questões de auto-relato de patologias tem sido freqüente 19,20 . Informações sobre asma autoreferida, especificamente o auto-relato de diagnóstico médico de asma, já foram utilizadas em vários estudos, incluindo aqueles conduzidos pelo Centers for Disease Control and Prevention (CDC), nos Estados Unidos 2,19.

Este estudo tem como objetivos investigar a associação entre a história de auto-relato de diagnóstico médico de asma, incluindo idade ao diagnóstico e o tempo de doença, e a presença de transtornos metais comuns.

\section{Métodos}

Trata-se de uma análise de dados seccionais da Fase 1 do Estudo Pró-Saúde (1999), um estudo de coorte entre funcionários técnico-administrativos do quadro efetivo de uma universidade no Estado do Rio de Janeiro, Brasil. A população de estudo constou de 4.030 participantes, que representaram $90,4 \%$ da população elegível para o estudo (4.459). Foram excluídos funcionários licenciados por motivos não relacionados à saúde ou cedidos a outras instituições.

Os dados foram obtidos mediante questionário autopreenchível, aplicado em grupos com apoio de pessoal treinado ${ }^{20}$. O questionário contemplou, entre outros aspectos, a história e a situação atual das condições sócio-econômicas, padrões de dieta, atividade física, consumo de tabaco e álcool, transtornos mentais comuns, morbidade auto-referida e tratamentos médicos, comportamentos e exposições com repercussões sobre a saúde.

A história de asma foi avaliada por intermédio das seguintes perguntas, incluídas em um bloco de perguntas sobre morbidade auto-referida, que incluíram também outras patologias como presença de hipertensão, artrose e diabetes: "Alguma vez um médico lhe informou que você teve ou tem asma ou bronquite asmática?" e "Com que idade um médico lhe informou pela primeira vez que você teve ou tem a condição ou doença?". Com base nessas perguntas avaliamos o tempo decorrido entre o diagnóstico médico e o momento do estudo, usando como pontos de corte 5 e 10 anos. Para a idade ao diagnóstico dividimos em quartis: < 3, 3-6, 7-19 e 20 ou mais anos de idade. Para a primeira pergunta, foram realizados dois estudos de confiabilidade teste-reteste: o primeiro, com duas semanas de intervalo entre aferições, como parte do estudo piloto da Fase 1 
(1999) do estudo de coorte, com uma amostra de 192 funcionários não efetivos; o segundo, com dois anos de intervalo, no qual compararam-se as respostas dadas na Fase $1 \mathrm{com}$ as respostas da Fase 2 (2001) do estudo, entre 3.147 funcionários que participaram das duas fases e exclusão dos casos incidentes. A avaliação da confiabilidade foi feita pelo coeficiente kappa.

A avaliação dos transtornos mentais comuns foi realizada com a versão resumida do General Health Questionnaire (GHQ-12), um instrumento bem estabelecido e amplamente utilizado em pesquisas nacionais e internacionais na área 21,22. Esse questionário autopreenchível foi validado na sua versão original e na sua versão brasileira, tendo, em ambos os casos, o Clinical Interview Schedule como padrão-ouro ${ }^{21,23}$. O ponto de corte utilizado para o questionário considerou cada item como presente ou ausente (0 ou 1), de acordo com o método do GHQ. Foram considerados casos de transtornos mentais comuns aqueles que foram positivos em pelo menos três itens do GHQ-12 24. O período de referência do GHQ-12 foram as duas semanas anteriores ao preenchimento do questionário.

A análise dos dados foi realizada com os pacotes estatísticos SPSS versão 8 (SPSS Inc., Chicago, Estados Unidos) e Stata versão 8 (Stata Corp., College Station, Estados Unidos). As seguintes variáveis foram avaliadas para potencial efeito de confundimento: sexo, idade (contínua), escolaridade (até 1 o grau incompleto, 1 o grau completo, 2o grau completo, 3 o grau completo ou mais), situação conjugal (casado/vive em união, solteiro, divorciado, viúvo) e renda familiar per capita (contínua). As questões referentes à asma autoreferida e transtornos mentais comuns foram avaliadas como variáveis dicotômicas (sim/não). As associações entre asma auto-referida, idade do diagnóstico de asma e tempo de diagnóstico de asma e transtornos mentais comuns foram examinadas por meio de regressão logística não condicional, com ajuste pelas variáveis sócio-demográficas que mostraram associação com asma e/ou transtornos mentais comuns a um nível de significância de 0,25 ou que, independentemente da associação encontrada, a literatura aponta como fator importante nesta associação, como foi o caso da idade. Foram utilizadas razões de prevalência (RP) brutas e ajustadas e seus respectivos intervalos de $95 \%$ de confiança.

\section{Resultados}

A confiabilidade teste-reteste do auto-relato de diagnóstico médico de asma foi substancial, tanto com duas semanas de intervalo (kappa $=0,75$; IC95\%: 0,61-0,90) quanto com intervalo de dois anos entre as perguntas (kappa $=0,72$; IC95\%: 0,68-0,76) (Tabela 1).

A prevalência do auto-relato de diagnóstico médico de asma ao longo da vida entre os 4.030 funcionários que responderam ao questionário foi de $11 \%(\mathrm{~N}=444)$; entre os que referiram asma, 39,7\% ( $\mathrm{N}=176)$ apresentavam transtornos mentais comuns. Com relação às variáveis sóciodemográficas, o auto-relato de asma associou-se com sexo feminino $(\mathrm{p}=0,001)$ e renda familiar per capita mais elevada $(p=0,01)$ (Tabela 2 ).

Na Tabela 3 observamos que, após ajuste por sexo, idade e renda familiar per capita, auto-relato de diagnóstico médico de asma associou-se a uma prevalência cerca de $40 \%$ mais elevada de transtornos mentais comuns $(\mathrm{RP}=1,37$; IC95\%: 1,21-1,55). Com relação à idade ao diagnóstico de asma, observou-se que aqueles cujo diagnóstico foi feito entre 7 e 19 anos apresentavam uma prevalência 53\% mais elevada de transtornos mentais comuns, enquanto entre aqueles cujo diagnóstico foi feito antes dos 7 anos de idade $\mathrm{e}$ depois dos 19 anos de idade o aumento na prevalência ficou entre 35 e 39\%. Para o tempo de diagnóstico de asma, verificou-se que aqueles com dez ou mais anos de diagnóstico apresentavam um risco menos elevado de transtornos mentais comuns do que aqueles cujo diagnóstico era mais recente.

\section{Discussão}

A prevalência do auto-relato de diagnóstico médico de asma nessa população foi de $11 \%$. Apesar da existência de estudos sobre a prevalência da asma 2,25, a utilização de diferentes critérios para a definição da asma torna difícil a comparação das prevalências de asma em diferentes partes do mundo. As recentes aplicações de métodos padronizados de medidas de prevalência da asma e dos sintomas de sibilos em adultos e crianças têm contribuído para se fazer comparações com maior segurança. No Brasil, a prevalência média de asma ativa na infância é em torno de $20 \% 2$, todavia, até o momento, não existem estudos sobre a prevalência de asma entre adultos na população geral.

A maioria dos estudos epidemiológicos sobre asma, sobretudo os que avaliam sua prevalência, utilizam questionários para sua realização. $\mathrm{O}$ uso de questionários depende da memória e 
Tabela 1

Confiabilidade teste-reteste do auto-relato de diagnóstico médico de asma entre os funcionários de uma universidade no Estado do Rio de Janeiro, Brasil, com duas semanas de intervalo e entre as Fases 1 (1999) e 2 (2001) do Estudo Pró-Saúde.

\begin{tabular}{|c|c|c|c|c|c|}
\hline & Sim & Não & Total & Kappa & IC95\% \\
\hline Duas semanas de intervalo & & & & 0,75 & $0,61-0,90$ \\
\hline Sim & 16 & 5 & 21 & & \\
\hline Não & 4 & 161 & 165 & & \\
\hline Total & 20 & 166 & 186 & & \\
\hline Dois anos de intervalo & & & & 0,72 & $0,68-0,76$ \\
\hline Sim & 241 & 71 & 312 & & \\
\hline Não & 88 & 2.747 & 2.835 & & \\
\hline Total & 329 & 2.818 & 3.147 & & \\
\hline
\end{tabular}

Tabela 2

Associação entre variáveis sócio-demográficas e auto-relato de diagnóstico médico de asma ao longo da vida entre funcionários de uma universidade no Estado do Rio de Janeiro, Brasil - Estudo Pró-Saúde (1999).

\begin{tabular}{|c|c|c|c|c|}
\hline \multirow[t]{2}{*}{ Variáveis sócio-demográficas } & \multirow[t]{2}{*}{ Total (N) } & \multicolumn{3}{|c|}{ Auto-relato de diagnóstico médico de asma } \\
\hline & & $\mathbf{N}$ & $\%$ & $p$ valor \\
\hline Sexo & & & & 0,001 \\
\hline Feminino & 2.210 & 288 & 13,0 & \\
\hline Masculino & 1.756 & 156 & 8,8 & \\
\hline Idade (anos) & & & & 0,608 \\
\hline$\leq 24$ & 41 & 5 & 10,9 & \\
\hline $25-34$ & 944 & 125 & 11,7 & \\
\hline $35-44$ & 1.527 & 188 & 11,0 & \\
\hline $45-54$ & 775 & 89 & 10,3 & \\
\hline$\geq 55$ & 234 & 37 & 11,2 & \\
\hline Anos de estudo & & & & 0,800 \\
\hline Até 1ㅇ grau incompleto & 232 & 31 & 11,8 & \\
\hline 1ㅇ grau completo & 587 & 58 & 9,0 & \\
\hline 2o grau completo & 1.264 & 150 & 10,6 & \\
\hline 3o grau completo ou mais & 1.450 & 202 & 12,6 & \\
\hline Situação conjugal & & & & 0,694 \\
\hline Casado/Vive em união & 2.069 & 231 & 11,2 & \\
\hline Solteiro/Viúvo/Divorciado & 1.324 & 143 & 11,6 & \\
\hline Renda familiar (salários mínimos) & & & & 0,010 \\
\hline$<3$ & 920 & 93 & 9,2 & \\
\hline $3-6$ & 1.213 & 158 & 11,5 & \\
\hline$>6$ & 1.170 & 178 & 13,2 & \\
\hline
\end{tabular}


Razões de prevalência (RP) brutas e ajustadas e respectivos intervalos de $95 \%$ de confiança (IC95\%) da associação entre auto-relato de diagnóstico médico de asma, idade de diagnóstico, tempo do diagnóstico e transtornos mentais comuns.

\begin{tabular}{|c|c|c|c|c|}
\hline \multirow[t]{2}{*}{ História de asma } & \multicolumn{4}{|c|}{ Transtornos mentais comuns } \\
\hline & $\mathbf{N}$ & $\%$ & RP bruta (IC95\%) & RP ajustada (IC95\%) \\
\hline \multicolumn{5}{|l|}{ Asma auto-referida $*, \star \star$} \\
\hline Não & 976 & 28,5 & 1,00 & 1,00 \\
\hline Sim & 176 & 40,5 & $1,41(1,25-1,60)$ & $1,37(1,21-1,55)$ \\
\hline \multicolumn{5}{|c|}{ Idade do diagnóstico de asma (anos) *,** } \\
\hline Sem diagnóstico de asma & 976 & 28,5 & 1,00 & 1,00 \\
\hline$<3$ & 45 & 42,5 & $1,50(1,18-1,86)$ & $1,39(1,10-1,74)$ \\
\hline $3-6$ & 39 & 38,2 & $1,34(1,04-1,72)$ & $1,35(1,05-1,73)$ \\
\hline 7-19 & 45 & 42,9 & $1,50(1,19-1,88)$ & $1,53(1,23-1,91)$ \\
\hline$\geq 20$ & 41 & 44,6 & $1,56(1,23-1,97)$ & $1,39(1,10-1,75)$ \\
\hline \multicolumn{5}{|c|}{ 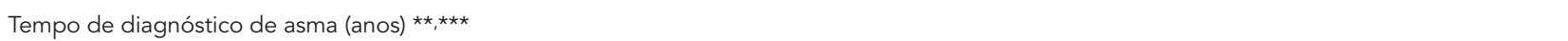 } \\
\hline Sem diagnóstico de asma & 976 & 28,5 & 1,00 & 1,00 \\
\hline$<5$ & 14 & 51,9 & $1,81(1,25-2,62)$ & $1,67(1,15-2,41)$ \\
\hline $5-9$ & 14 & 53,4 & $1,88(1,31-2,70)$ & $1,68(1,20-2,35)$ \\
\hline$\geq 10$ & 141 & 40,1 & $1,40(1,22-1,61)$ & $1,38(1,21-1,59)$ \\
\hline
\end{tabular}

dos interesses peculiares dos indivíduos em relação aos temas de investigação. Os questionários padronizados vêm sendo aplicados a crianças ${ }^{2} \mathrm{e}$ adultos ${ }^{17}$ para avaliação de grandes populações, por conta das dificuldades operacionais na realização de testes de hiper-reatividade brônquica nessa população. Em nosso estudo, as respostas à pergunta sobre auto-relato de diagnóstico médico foram avaliadas por dois estudos de confiabilidade teste-reteste; o primeiro com duas semanas de intervalo e o segundo com dois anos de intervalo entre as aferições. Em ambos os estudos a confiabilidade foi considerada substancial, sugerindo estabilidade da resposta.

No presente estudo, observou-se que a distribuição da asma auto-referida estava associada diretamente à renda familiar per capita $(\mathrm{p}=0,01)$. A relação entre renda e asma é variável entre os estudos 4,26, fato que sugere relações complexas entre asma e fatores sócio-econômicos. Estudos realizados nos anos 60 e 704 sugerem que a asma era mais comum em crianças de famílias de maior renda. Tais achados estão de acordo com a "hipótese higiênica", que sugere uma associação entre asma e melhora das condições de higiene, particularmente em países desenvolvidos 5 . Uma outra avaliação sugere que o diagnóstico da asma pode ser mais freqüente nas classes sociais mais altas devido à maior facilidade de acesso aos ser- viços de saúde ou a um diagnóstico de asma superestimado nessa população 4,27 .

Os resultados encontrados neste estudo mostram que existe associação entre auto-relato de diagnóstico médico de asma e transtornos mentais comuns. A prevalência de transtornos mentais comuns em indivíduos com história de auto-relato de asma ao longo da vida foi de $39,7 \%$, sendo a associação ajustada estatisticamente significante (RP = 1,37; IC95\%: 1,21-1,55). Esse achado é consistente com os descritos na literatura, que mostram um risco mais elevado de transtornos mentais entre indivíduos com diagnóstico de asma, estejam eles em tratamento ou não 8,12,28

Indivíduos com menos de dez anos de diagnóstico de asma apresentaram uma prevalência de transtornos mentais comuns aproximadamente $85 \%$ mais elevada do que aqueles sem diagnóstico de asma. Semelhante achado sugere uma inadequação ao novo status de doença. Não encontramos, até o momento, nenhum outro estudo que tenha avaliado essa associação.

Uma das limitações do presente estudo se refere à natureza seccional do desenho, no qual as observações foram realizadas em uma única oportunidade, não permitindo estabelecer de modo definitivo a seqüência temporal entre asma e transtornos mentais comuns. O fato, con- 
tudo, de a asma ser uma doença crônica e seu relato neste estudo se referir à história de asma ao longo da vida, e o período de referência dos transtornos mentais comuns ser as duas semanas anteriores, reforçam a hipótese do estudo de que a asma, como doença crônica, estaria associada a um aumento na prevalência de depressão e/ou ansiedade. Outra limitação deste estudo foi a utilização somente da pergunta sobre a história de um diagnóstico médico de asma sem a realização de exame complementar para corroborar o diagnóstico. Porém, a realização de tais exames em grandes populações envolve logística complexa, nem sempre ao alcance dos pesquisadores. Estudos utilizando questionários autopreenchíveis, com perguntas sobre história de diagnóstico médico de doenças ao longo da vida, têm sido considerados válidos na

\section{Resumo}

Este estudo investiga a associação entre história de asma e transtornos mentais comuns em funcionários de uma universidade no Rio de Janeiro, Brasil. Dados seccionais da Fase 1 (1999) de um estudo de coorte (Estudo Pró-Saúde) foram coletados entre 4.030 funcionários. A asma foi avaliada mediante auto-relato de diagnóstico médico e a presença de transtornos mentais comuns pelo General Health Questionnaire (GHQ-12). Modelos lineares generalizados foram utilizados para o cálculo de razões de prevalência. A prevalência de asma foi de $11 \%$ (444), dos quais 39,7\% (176) apresentavam transtornos mentais comuns. História de asma associou-se com renda mais elevada $(p=0,01) e$ sexo feminino $(p=0,001)$. A análise ajustada por sexo, idade e renda mostrou associação entre história de asma e transtornos mentais comuns ( $R P=1,37$; IC95\%: 1,22-1,55), e aqueles com menos de dez anos de diagnóstico apresentaram uma prevalência de transtornos mentais comuns mais elevada do que aqueles sem diagnóstico de asma (RP = 1,88; IC95\%: 1,32-2,70). Esses achados reforçam a necessidade de atenção por parte das equipes multidisciplinares dos aspectos emocionais dos pacientes com asma, em especial daqueles com menos tempo de convivência com a doença.

Asma; Transtornos Mentais; Estudos de Coortes; Questionários identificação de morbidade física nessas populações 18,19 .

Deve-se ressaltar, também, que o estudo foi realizado numa amostra de funcionários públicos com características particulares, fator que limita a possibilidade de seus achados serem generalizados para a população geral. Os achados do presente estudo, no entanto, são consistentes com os da literatura internacional e reforçam a pertinência de estudos longitudinais que avaliam os efeitos de longo prazo de asma.

$\mathrm{O}$ conhecimento sobre a associação entre asma e transtornos mentais comuns deve sensibilizar as equipes multidisciplinares para as questões de caráter emocional de seus pacientes, e subsidiar ações voltadas para a prevenção de transtornos mentais comuns em pacientes com asma.

\section{Colaboradores}

K. T. Nogueira revisou a literatura, realizou a análise estatística dos dados e participou da interpretação e discussão dos resultados. C. S. Lopes participou do planejamento e implementação do Estudo Pró-Saúde e orientou a revisão bibliográfica, análise de dados e interpretação e discussão dos resultados. E. Faerstein participou do planejamento e implementação do Estudo Pró-Saúde e interpretação e discussão dos resultados. Todos os autores trabalharam na elaboração final do artigo.

\section{Agradecimentos}

A toda a equipe do Estudo Pró-Saúde pela participação, apoio e cooperação durante as diferentes etapas desse trabalho. 


\section{Referências}

1. World Health Organization. Bronquial asthma 2004. http://www.who.int/mediacentre/factsheets/ fs206/en (acessado em Mar/2005).

2. Worldwide variations in the prevalence of asthma symptoms: the International Study of Asthma and Allergies in Childhood (ISAAC). Eur Respir J 1998; 12:315-35.

3. III Consenso Brasileiro no Manejo da Asma 2002. J Pneumol 2002; 28 Suppl 1.

4. Global Initiative for Asthma. Global initiative for asthma management and prevention. Bethesda: U.S. Department of Health and Human Services, National Institutes of Health; 2002.

5. Czeresnia D. The hygienic hypothesis and transformations in etiological knowledge: from causal ontology to ontogenesis of the body. Cad Saúde Pública 2005; 21:1168-76.

6. Miller BD. Depression and asthma: a potential lethal mixture. J Allergy Clin Immunol 1987; 80:481-6.

7. Rimington LD, Davies DH, Lowe D. Relationship between anxiety, depression, and morbidity in adult asthma patients. Thorax 2001; 56:266-71.

8. Wright RJ, Rodriguez M, Cohen S. Review of psychosocial stress and asthma: an integrated biopsychosocial approach. Thorax 1998; 53:1066-74.

9. Gillaspy SR, Hoffa L, Mullins LL, Vanpelt JC, Chaney JM. Psychological distress in high-risk youth with asthma. J Pediatr Psychol 2002; 27:363-71.

10. Centanni S, Di Marco F, Castagna F, Boveri B, Casanova F, Piazzini A. Psychological issues in the treatment of asthmatic patients. Respir Med 2000; 94:742-9.

11. Castro RC, Santos NO, Morettom LT. Depressão e eventos de vida relacionados à asma grave. Rev Bras Alergia Imunopatol 2001; 24:204-11.

12. Machado AS, Machado DT, Portela PG. Freqüência de depressão em pacientes ambulatoriais com asma moderada e grave. Rev Bras Alergia Imunopatol 2000; 23:90-7.

13. Myers JK, Weissman M, Ticheler GE. Six-month prevalence of psychiatric disorders in three communities. Arch Gen Psychiatry 1994; 41:959-70.

14. Coyne JC, Fechener-Bates S, Schwenk TL. Prevalence, nature, and comorbidity of depressive disorders in primary care. Gen Hosp Psychiatry 1994; 16:267-76.
15. Fletcher CM, Tinker CM. Chronic bronchitis. A further study of simple diagnostic methods in a working population. Br Med J 1961; 27:1491-8.

16. Ferris BG. Epidemiology standardization project (American Thoracic Society). Am Rev Respir Dis 1978; 118(6 Pt 2):1-120.

17. Burney PG, Laitinen LA, Perdrizet S, Huckauf H, Tattersfield AE, Chin S, et al. Validity and repeatability of IUATLD (1984) Bronchial Symptoms Questionnaire: an international comparison. Eur Respir J 1989; 2:940-5.

18. Torén K, Brisman J, Jarvholm B. Asthma and asthma-like symptoms in adults assessed by questionnaires. A literature review. Chest 1993; 104:600-8.

19. Cheng Y, Macera CA, Davis RD, Barbara A, Troped PJ, Blair SN. Physical activity and self-reported, physician-diagnosed osteoarthritis is physical activity a risk factor? J Clin Epidemiol 2000; 53: 315-22.

20. Faerstein E, Lopes CS, Valente K, Sole-Plá MA, Ferreira MB. Pré-testes de um questionário multidimensional autopreenchível: a experiência do Estudo Pró-Saúde UERJ. Physis 1999; 9:117-30.

21. Mari JJ, Willians PA. Comparison of the validity of two psychiatric screening questionnaires (GHQ12 and SRQ-20) in Brazil, using Relative Operating Characteristic (ROC) analysis. Psychol Med 1985; 15:651-9.

22. Weich S, Lewis G. Poverty, unemployment, and common mental disorders: population based cohort study. BMJ 1998; 317:115-9.

23. Goldberg DP, Blackwell B. The detection of psychiatric illness by questionnaire. London: Oxford University Press; 1970. (Maudsley Monograph, 21).

24. Goldberg DP, Williams P. The user's guide to the General Health Questionnaire. Nelson: Windsor; 1988.

25. Strachan DP. The epidemiology of childhood asthma. Allergy 1999; 54:7-11.

26. Burr ML. Epidemiology of asthma. Monogr Allergy 1993; 31:80-92.

27. Newacheck PW, McMamanus MA, Fox HB. Prevalence and impact of chronic illness among adolescent. Am J Dis Child 1999; 145:1367-73.

28. Perna P, Bertani A, Politi E. Asthma and panic attacks. Biol Psychiatry 1997; 42:625-30.

Recebido em 02/Dez/2005

Versão final reapresentada em 01/Nov/2006

Aprovado em 03/Jan/2007 